\title{
GAME OF THRONES E A CIÊNCIA DAS REDES
}

Network of Thrones

\author{
Andrew Beveridge e Jie Shan ${ }^{1}$ \\ Lindolfo Sancho (tradutor) ${ }^{2}$
}

Game of Thrones, o sucesso internacional da HBO adaptado da saga literária de fantasia épica "As crônicas de Gelo e Fogo", de George R. R. Martin, apresenta enredos entrelaçados e dezenas de personagens. Com tantas pessoas para acompanhar nessa saga extensa, pode ser um desafio entender completamente as dinâmicas entre os personagens.

Para desmistificar esta saga, nos voltamos para a Ciência das Redes, um ramo novo e em evolução da Teoria dos Grafos ${ }^{3}$ que une tradições de diferentes disciplinas, incluindo sociologia, economia, física, ciência da computação, e matemática. A ciência das redes tem sido amplamente aplicada nas ciências em geral, nas ciências sociais, humanidades, e em contextos industriais.

Neste artigo, realizamos uma análise de rede de Game of Thrones para esclarecer os complexos relacionamentos entre os personagens e suas posições no futuro do enredo (mas prometemos: sem spoilers!).

1 Agradecemos aos autores, Andrew Beveridge e Jie Shan, por autorizarem a tradução do artigo para a Pensata. A versão original, Network of Thrones, foi publicada na revista Math Horizons, 23 (DOI: 10.4169/mathhorizons.23.4.18), sendo publicado aqui com permissão de Taylor \& Francis Ltd. em nome da Associação Matemática da América.

Agradecemos também à Felipe Fiamozzini, que realizou a revisão do texto com atenção especial aos termos utilizados na ciência das redes.

2 Lindolfo Sancho é mestrando em Ciênicas Sociais pela Universidade Federal de São Paulo (EFLCH/UNIFESP). E-mail: lindolfocampos@gmail.com

${ }^{3}$ Nota do tradutor: um grafo é composto por dois conjuntos. Um conjunto de vértices, ou nós, (V), e um conjunto de arestas (E), chamados arcos quando possuem um vértice em cada ponta. 


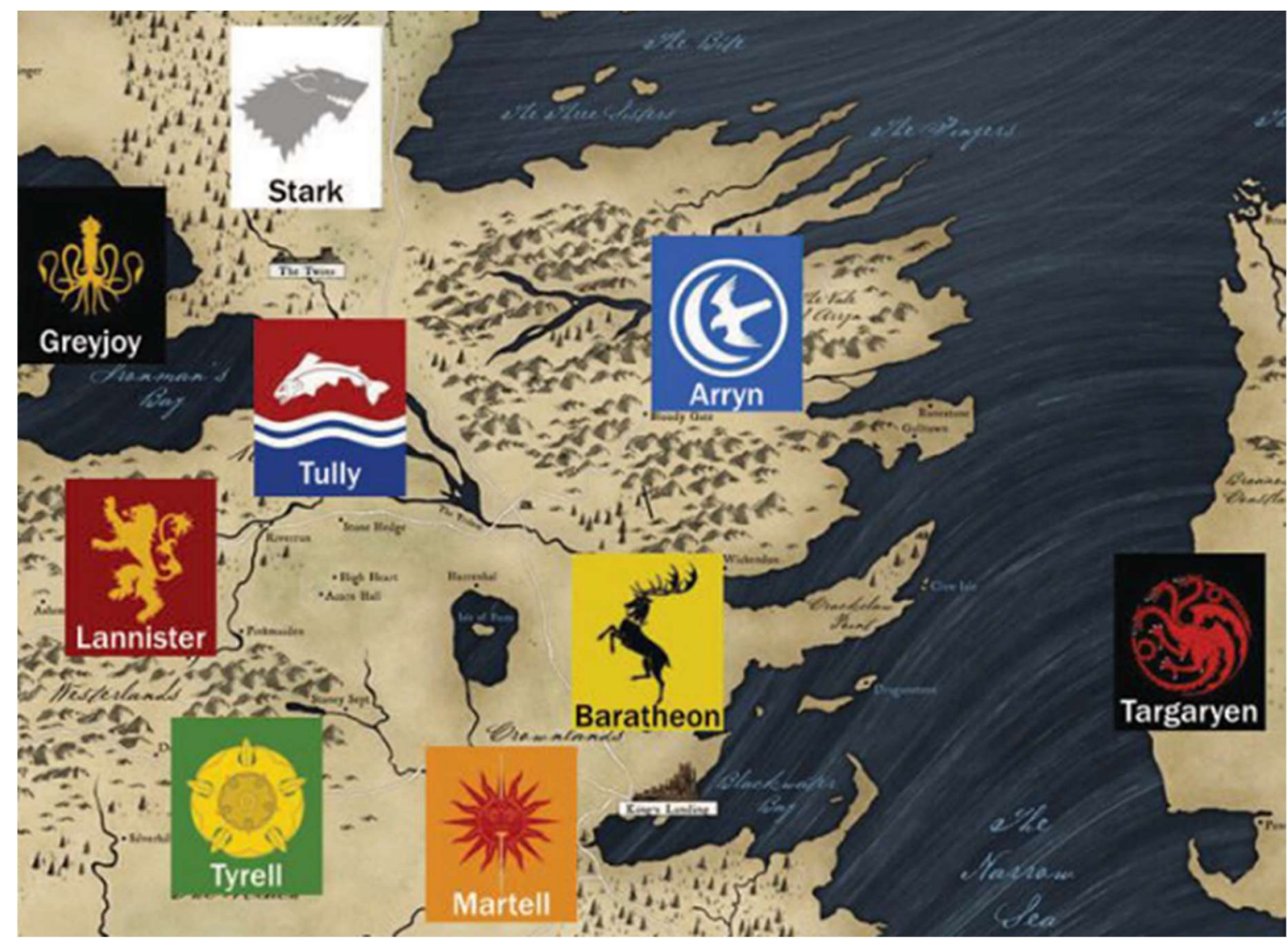

Figura 1 - O mundo de Game of Thrones: Westeros, o Mar Estreito e Essos (da esquerda para direita). Os brasões representam as localidades das casas nobres no começo da saga.

Primeiro, uma rápida introdução à Game of Thrones: Westeros e Essos, separados pelo Mar Estreito, abrigam uma série de famílias nobres (FIGURA 1). A narrativa começa em tempos de paz, com todas as casas nobres unificadas sob o jugo do rei Robert Baratheon, que controla o Trono de Ferro. Prematuramente, o rei Robert morre em um acidente de caça, e o jovem e cruel príncipe Joffrey ascende ao trono com o apoio dos Lannister, sua casa materna. Entretanto, a legitimidade do príncipe, e mesmo sua identidade, é seriamente questionada por todo o reino. Como resultado, começa a guerra com múltiplos pretendentes ao Trono de Ferro.

Seja por causa ou circunstância, personagens das muitas famílias nobres se lançam em jornadas longas e penosas. Entre as casas há a honorável família Stark (Eddard, Catelyn, Robb, Sansa, Arya, Bran, e Jon Snow), os suntuosos Lannister (Twyin, Jaime, Cersei, Tyrion, e Joffrey), os desprezados Baratheon (liderados pelo irmão de Robert, Stannis) e a exilada Daenerys, último membro da uma vez poderosa casa Targaryen. 


\section{REVISTA PENSATA | V.7 N.1}

\section{DEZEMBRO DE 2018}

\section{A REDE SOCIAL}

Nossa primeira tarefa é transformar o mundo de Game of Thrones em uma rede social. Nossa rede, exibida na figura 2, possui um conjunto de vértices $\mathbf{V}$ e arestas $\mathbf{E}$. Os 107 vértices representam os personagens, incluindo Senhoras e Lordes, soldados e mercenários, conselheiros e consortes, aldeões e selvagens. Os vértices são conectados por 353 arestas ponderadas ${ }^{4}$, nos quais valores maiores correspondem a relacionamentos mais fortes entre os personagens conectados.

Nós geramos as arestas com base no terceiro livro da saga, "A Tormenta de Espadas". Optamos por esse volume porque a narrativa principal está amadurecida, com os personagens distanciados geograficamente e enredados em seus próprios círculos sociais. Utilizamos uma técnica de parsing ${ }^{5}$ para avaliar o ebook, incrementando o peso das arestas entre dois personagens quando seus nomes (ou apelidos) apareciam em uma distância menor que 15 palavras entre um e outro. Depois, realizamos algumas validações e retoques manualmente. Note-se que um arco entre dois personagens não significa necessariamente que sejam amigos - isso apenas indica que eles interagem, falam um do outro, ou são mencionados juntos.

\footnotetext{
${ }^{4}$ Nota do tradutor: Um grafo é ponderado, ou valorado, quando suas arestas (E) possuem um valor.

5 O termo original, "parse", é uma técnica específica de programação que consiste em utilizar funções para analisar relações entre as palavras de maneira automática.
} 


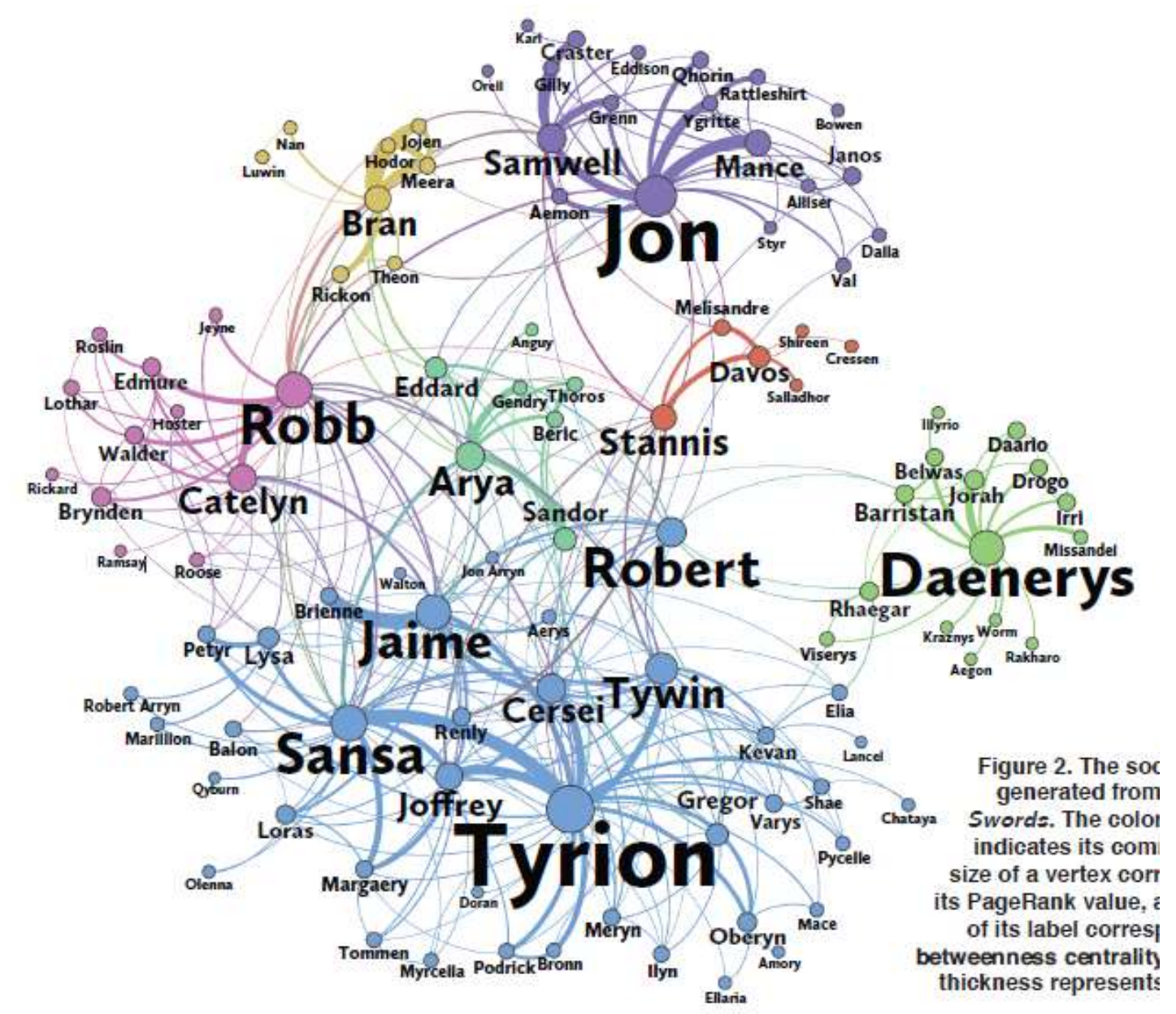

Figura 2 - A rede social gerada a partir de a Tormenta de Espadas. A cor dos vértices indicam as comunidades. O Tamanho de um vértice corresponde ao seu valor de PageRank e o tamanho dos nomes corresponde à centralidade de intermediação. A espessura de uma aresta representa seu peso

A complexidade da estrutura da nossa rede reflete os enredos emaranhados da história. Notavelmente, observamos duas características encontradas em muitas redes na vida real. Primeiro, a rede contém múltiplas sub-redes mais densas, unidas por uma rede global menos densa de arestas. Segundo, elas são organizadas em torno de um subconjunto de personagens altamente influentes, tanto localmente quanto globalmente. Agora descreveremos como quantificar essas observações usando as ferramentas analíticas da ciência das redes.

\section{DETECÇÃo DE COMUNIDADES}

$O$ traçado $\mathrm{e}$ as cores da rede na figura 2 claramente identifica sete comunidades. Os Lannister de Porto Real, o exército de Robb, Bran e amigos, Arya e 


\section{REVISTA PENSATA | V.7 N.1}

\section{DEZEMBRO DE 2018}

companheiros, Jon Snow e o extremo Norte, o exército de Stannis, e Daenerys e os povos exóticos de Essos.

Notavelmente, essas comunidades foram identificadas apenas a partir da estrutura da rede, como explicamos abaixo.

Queremos dividir a rede em comunidades coerentes, isso significa que há muitas arestas dentro das comunidades e poucas arestas entre as comunidades. Detectamos nossas comunidades na rede ao usar uma métrica global chamada modularidade. Assumindo que $W_{i j} \geq 0 W_{i j} \geq 0$ indica o valor da aresta entre os vértices i e j, onde $W_{i j}=0 W_{i j}=0$ indica que não há ligações, $K i=\sum j \in v^{W i j} K i=\sum j \in v^{W i j}$ indica o grau ponderado do vértice ${ }^{i i}$. Intuitivamente, a modularidade $Q$ compara uma dada rede com uma outra rede hipotética que contenha os mesmos graus calculados para todos os vértices, mas na qual todas as arestas são reorganizadas aleatoriamente. Esta rede hipotética não deveria ter nenhuma comunidade, o que a torna uma rede de referência para comparação.

Suponha que os vértices $i i$ e $i j$ pertençam à mesma comunidade $C C$. Esperaríamos que ${ }^{W i j W i j}$ fosse pelo menos tão grande quanto o número de arestas entre eles em nossa rede de referência. Através de análise combinatória pode-se demonstrar que o número esperado dessas arestas aleatórias é $\mathrm{KiKj} / 2 \mathrm{mKiKj} / 2 m$, onde ${ }^{M M}$ é o número total de arestas na rede. Somando todos os vértices em uma comunidade ${ }^{C C}$ temos:

$\sum_{i, j \in C}\left(W i j-\frac{K i K j}{2 m}\right) \geq 0$

Se ${ }^{C C}$ não for realmente uma comunidade então esta quantidade será negativa. A modularidade $Q Q$ de cada comunidade $C_{1, \ldots, \ldots}, C_{1} C_{1, \ldots, \ldots} C_{1}$ é:

$$
Q=\frac{1}{2 m} \sum_{k=1}^{l} \sum_{i, j \in C k}\left(W i j-\frac{K i K j}{2 m}\right)
$$

onde normalizamos essa quantidade para que $-1 \leq Q \leq 1-1 \leq Q \leq 1$.

Nosso objetivo é dividir os vértices em comunidades para maximizar $Q Q$. Encontrar essa divisão é difícil, mesmo computacionalmente, então usamos um 


\section{REVISTA PENSATA | V.7 N.1}

algoritmo de aproximação rápida chamado método Louvain ${ }^{6}$, que essencialmente determina o número de comunidades que maximizam $Q$.

Em nosso caso, descobrimos as sete comunidades na figura 2. A comunidade de Porto Real corresponde a $37 \%$ da rede. Quando realizamos identificação de comunidades nessa importante sub-rede, obtemos quatro comunidades.

Uma versão em alta-resolução da figura 2 e a rede de sub-comunidades de Porto Real pode ser encontrada em www.maa.org/math-orizons-supplements.

\section{MEDIDAS DE CENTRALIDADE}

A ciência das redes também pode identificar vértices importantes. Uma pessoa pode desempenhar um papel importante de múltiplas maneiras. Por exemplo, ela pode ser bem conectada, ocupar uma localização central na rede, ou estar em uma posição privilegiada para dispersar informações ou influenciar outras pessoas. A figura 3 mostra a importância de 14 personagens proeminentes, de acordo com seis medidas de centralidade, as quais explicamos abaixo.

${ }^{6} \mathrm{O}$ método Louvain é um procedimento de detecção de comunidades em grandes redes. Este método revela hierarquias entre comunidades, permitindo a detecção de sub-comunidades, e comunidades cada vez menores dentro de grandes redes. Uma descrição rápida deste método pode ser encontrada em: https://perso.uclouvain.be/vincent.blondel/research/louvain.html (acesso em 15/06/2019). 

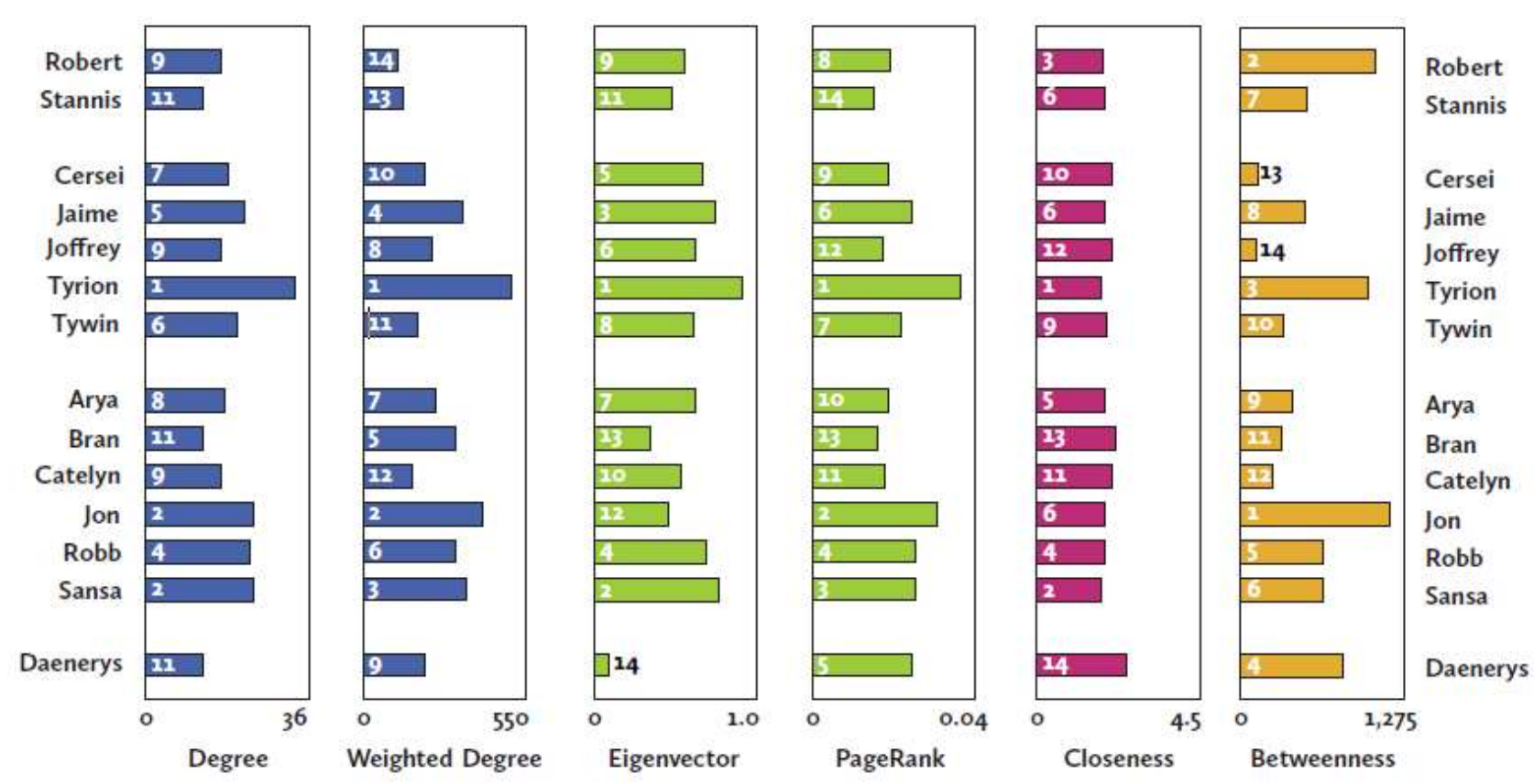

Figura 3 - Medidas de centralidade para a rede, valores maiores correspondem a maior importância dos personagens, exceto para centralidade de proximidade, onde valores mais baixos são melhores. Os números nas barras indicam a posição desses personagens.

Centralidade de grau é o número de arestas que convergem em um vértice. Centralidade de grau ponderada é similarmente definida pela soma dos pesos das arestas convergentes.

Em nossa rede, centralidade de grau mede o número de conexões entre os personagens, enquanto a centralidade de grau valorada mede o número de interações entre esses personagens.

Centralidade de autovetor é a centralidade de grau ponderada com um retorno ao próprio vértice: um vértice é impulsionado por estar conectado com vértices importantes. A importância ${ }^{X i X i}$ do vértice ${ }^{i i}$ é o valor somado da importância de seus vértices vizinhos: $X i=\sum j \in v^{W i j X j} X i=\sum j \in v^{W i j X j}$ para cada $i \in V i \in V$. Solucionar o sistema linear resultante nos dá a centralidade de autovetor. (Este nome vem da álgebra linear: na verdade, encontramos o autovetor para um autovalor $\lambda=1 \lambda=1 \mathrm{da}$ matriz $W W$ com entradas $W i j W i j$.)

Vamos comparar a centralidade de grau e a centralidade de autovetor da nossa rede. $O$ falecido Rei Robert recebe um grande impulso: Ele tem apenas 18 conexões, 


\section{REVISTA PENSATA | V.7 N.1}

\section{DEZEMBRO DE 2018}

mas metade delas com outros personagens proeminentes! A maioria dos personagens principais são beneficiados pela importância de seus vértices vizinhos, por estarem diretamente envolvidos nas intrigas políticas e no vasto tumulto militar dominando o reino. As exceções são personagens isolados da ação principal: Bran (supostamente morto e em fuga), Jon Snow (marginalizado no extremo Norte), e Daenerys (exilada para além do Mar Estreito).

PageRank é outra variação neste tema. Esta medida foi a ideia inicial por trás do mecanismo de busca Google, de Brin e Paige. Cada vértice possui uma importância própria $\beta \geq 0$ junto a uma importância adquirida de seus vizinhos. Diferentemente da centralidade de autovetor, um vértice não ganha toda atenção por causa da importância total de seus vizinhos. Em vez disso, a importância desse vizinho é igualmente dividida entre suas conexões diretas. Em outras palavras, um vértice de grau muito alto distribui apenas uma fração de sua importância a cada um de seus vizinhos.

O PageRank ${ }^{Y i Y i}$ do vértice ${ }^{i i}$ é dado por:

$Y i=\alpha \sum_{j \in V} \frac{W j i}{K j} Y i+\beta$

onde $\alpha+\beta=1 \alpha+\beta=1$ e $\alpha, \beta \geq 0 \alpha, \beta \geq 0$. Pesquisadores normalmente utilizam $\beta=0.15 \beta=0.15$ para encontrar um balanço efetivo entre importância própria e importância impulsionada por vizinhos.

PageRank não penaliza nossos três personagens distantes e na verdade tem o efeito contrário em Daenerys. De fato, a hierarquização por PageRank é quase idêntica à hierarquização pela centralidade de grau, exceto por Daenerys que passa da $12^{\circ}$ posição para quinto lugar. Assim, PageRank identifica corretamente a carismática Daenerys como um dos jogadores mais importantes, ainda que ela tenha poucas conexões.

Isso nos leva a duas medidas de centralidade cujas definições adotam uma visão mais global da rede. A centralidade de proximidade de um vértice é a distância média de um vértice a todos os outros vértices. (Diferentemente das outras medidas de centralidade, valores menores correspondem a maior importância.) Os valores de proximidade em nossa lista de personagens principais é bastante comprimida, exceto 


\section{REVISTA PENSATA | V.7 N.1}

pela distante Daenerys. Entretanto, Tyrion e Sansa obtém uma pequena vantagem sobre os outros.

A última medida de centralidade é a mais sutil. A centralidade de proximidade de um vértice mede a frequência com que esse vértice se encontra em atalhos entre outros pares de vértices. Matematicamente, a intermediação ${ }^{Z i Z i}$ do vértice ${ }^{i i}$ é:

$$
Z i=\sum_{j, k \in V} \frac{\sigma j k(i)}{\sigma j k}
$$

Onde $\sigma j k \sigma j k$ é o número de $(j, k)(j, k)_{\text {-atalhos } \mathrm{e}}$ $\sigma j k(i) \sigma j k(i)$ é o número desses $(j, k)(j, k)_{-}$atalhos que passam pelo vértice ${ }^{i i}$. Um vértice que aparece em muitos atalhos é um intermediário de informações na rede: comunicação eficiente entre diferentes partes da rede vai frequentemente passar por tal vértice. Tais conectores podem ser altamente influentes ao se inserirem nos assuntos de outras partes.

Centralidade de intermediação oferece uma classificação distinta dos personagens. Apenas nesta medida Tyrion não aparece no topo: ele é o terceiro, atrás de Jon Snow (graças a sua ligação tanto com a Casa Stark quanto com os remotos habitantes do Norte) e Robert Baratheon (a única pessoa diretamente conectada com todas as quatro Casas nobres dos personagens principais). Enquanto isso, Daenerys é elevada à quarta posição (sua melhor classificação) por causa da natureza hub-andspoke ${ }^{7}$ da eclética comunidade de Essos.

Não há uma única medida de centralidade correta para uma rede. Cada medida oferece informações complementares e levar cada uma em consideração pode ser bastante revelador. Em nossa rede, três personagens se destacam de forma constante: Tyrion, Jon Snow, e Sansa. Atuando como Mão do Rei, Tyrion é empurrado para o centro das maquinações políticas da capital. Nossa análise sugere que ele é o verdadeiro protagonista do livro.

Enquanto isso, Jon Snow é distintamente posicionado na rede, com ligações com nobres, com a milícia da Patrulha da Noite, e com os selvagens de além da

${ }^{7}$ Nota do tradutor: Hub-and-spoke é uma expressão adotada do sistema de transporte aéreo onde um grande aeroporto (hub) opera como centro de distribuição de rotas menores entre rotas de longa-distância (spoke). Isso reflete a posição central de Daenerys em Essos. 


\section{REVISTA PENSATA | V.7 N.1}

\section{DEZEMBRO DE 2018}

Muralha. A grande surpresa talvez seja a proeminência de Sansa Stark, uma cativa de facto em Porto Real. Entretanto, outros personagens reconhecem seu valor como uma herdeira Stark, a usando repetidas vezes como um peão em suas estratégias pelo poder. Se desenvolver sua astúcia, ela pode capitalizar sobre sua importância na rede para grande efeito.

Robert e Daenerys, por sua vez, se destacam por se classificarem bem em certas medidas de centralidade. Eles oferecem um claro contraponto um ao outro e retornam nossa atenção para o Trono de Ferro. A memória de Robert unifica a rede esmigalhada do passado recente, enquanto Daenerys vai certamente perturbar a rede atual quando retornar a Westeros em busca do Trono de Ferro.

\section{UMA VIDA EM REDE}

Nós visitamos os reinos de Westeros e Essos para apresentar as ferramentas básicas da ciência das redes. Realizamos uma análise empírica de nossa rede, encontrando comunidades e identificando pessoas influentes. Nossa análise de rede confirma algumas expectativas e oferece novas perspectivas sobre essa saga ricamente imaginada. Nós consideramos uma aplicação fantasiosa da ciência das redes para oferecer uma visão atraente de suas possibilidades. Usos mais sérios estão por toda parte, e a ciência das redes promete se tornar inestimável na compreensão de nossa moderna vida em rede.

\section{LEITURA ADICIONAL}

[1] A.-L. Barabási, Network Science. barabasilab.neu.edu/networksciencebook/downIPDF.html.

[2] D. Easley, J. Kleinberg. Networks, Crowds and Markets. Cambridge University Press, Cambridge,2010.

[3] S. Fortunato. Community detection in graphs. Physics Reports 486 nos. 3-5 (2010) 75-174.

[4] M. Newman, Networks: An Introduction. Oxford University Press, Oxford, 2010. 